\title{
Decreased H3K27 and H3K4 trimethylation on mortal chromosomes in distributed stem cells
}

\author{
YH Huh ${ }^{1,2}$ and JL Sherley,
}

The role of immortal DNA strands that co-segregate during mitosis of asymmetrically self-renewing distributed stem cells (DSCs) is unknown. Previously, investigation of immortal DNA strand function and molecular mechanisms responsible for their nonrandom co-segregation was precluded by difficulty in identifying DSCs and immortal DNA strands. Here, we report the use of two technological innovations, selective DSC expansion and establishment of H2A.Z chromosomal asymmetry as a specific marker of 'immortal chromosomes', to investigate molecular properties of immortal chromosomes and opposing 'mortal chromosomes' in cultured mouse hair follicle DSCs. Although detection of the respective suppressive and activating H3K27me3 and H3K4me3 epigenetic marks on immortal chromosomes was similar to randomly segregated chromosomes, detection of both was lower on mortal chromosomes destined for lineage-committed sister cells. This global epigenomic feature of nonrandom co-segregation may reveal a mechanism that maintains an epigenome-wide 'poised' transcription state, which preserves DSC identity, while simultaneously activating sister chromosomes for differentiation.

Cell Death and Disease (2014) 5, e1554; doi:10.1038/cddis.2014.522; published online 4 December 2014

Distributed stem cells (DSCs) are responsible for the continuous replenishment of the short-lived differentiating cells of renewing tissues. ${ }^{1-9}$ DSCs accomplish this role by their unique asymmetric self-renewal, a cell division program that preserves stem cell phenotype with simultaneous production of cells committed to tissue-specific differentiation lineages. ${ }^{2,3}$ Tightly associated with asymmetric self-renewal, DSCs adopt a nonrandom form of mitotic chromosome segregation defined by continuous co-segregation of the set of chromosomes with the older template DNA strands to asymmetrically dividing DSCs. ${ }^{7,10,11}$ These oldest DNA strands in asymmetrically self-renewing DSCs are called 'immortal strands', ${ }^{1}$ and the chromosomes bearing them are called 'immortal chromosomes'. ${ }^{6}$ Immortal DNA strand cosegregation has been detected in an increasingly diverse range of tissues and vertebrate species. ${ }^{7,12-24}$

Nonrandom sister chromatid segregation (that is, cosegregation of immortal chromosomes) was originally proposed as a mechanism that could limit DSC gene mutations that arise from DNA replication errors. ${ }^{1}$ More recently, it has been suggested to have a role in preserving the stemness phenotype of DSCs during asymmetric self-renewal divisions, ${ }^{25}$ and accumulated damage to long-lived immortal DNA strands in DSCs has been proposed as a mechanism of tissue aging. ${ }^{2}$ However, neither the exact cellular function(s) of immortal chromosomes nor the mechanisms responsible for their nonrandom co-segregation are known.

Previously, investigations of the function of nonrandom cosegregation of immortal chromosomes and the responsible molecular mechanisms were largely precluded because of difficulty in obtaining DSCs in the quantity and purity required for meaningful molecular analyses and the lack of direct assays for detection of immortal DNA strands or their immortal chromosomes. Toward enabling molecular investigations of immortal DNA strands, we addressed the DSC scarcity problem by developing a general method for selective ex vivo expansion of diverse DSCs. This method is based on suppressing the asymmetric cell kinetics (suppression of asymmetric cell kinetics; 'SACK') of DSCs. ${ }^{4,5,7}$ The DSC fraction of cultures of the SACK-derived, adult mouse hair follicle DSCs used in the presented studies is estimated to be $\geqslant 44 \%$. The derivation of these clonal DSC strains has been described previously. ${ }^{7}$ In brief, they were derived from cell cultures outgrown from isolated mouse whisker follicles incubated in medium supplemented with the SACK agent xanthine $(X n)$. Before culture, whisker follicles were first cleared of all external cellular material and then slit with a small incision to allow follicle cells to divide and escape. Subsequently, cell strains with $\mathrm{Xn}$-dependent growth were clonally derived. Their DSCs properties include purinedependent asymmetric self-renewal, purine-dependent nonrandom sister chromatid segregation, long-term self-renewal, and production of multiple differentiated cell types of the skin and hair follicles. ${ }^{7}$

For much of their history, immortal DNA strands were mainly detected with retrospective assays based on their labeling properties with DNA base analogs. ${ }^{7,12-15,17-24}$ With one recent exception, ${ }^{21,24}$ these assays require chromosome

\footnotetext{
${ }^{1}$ The Adult Stem Cell Technology Center, LLC, Boston, MA, USA and ${ }^{2}$ Division of Electron Microscopic Research, Korea Basic Science Institute, 169-148 Gwahak-ro, Yuseong-gu, Daejeon, Korea

*Corresponding author: JL Sherley, The Adult Stem Cell Technology Center, LLC, PO Box 301179, Boston 02130, MA, USA. Tel: 6179906819 ; E-mail: jlsherley @ gmail.com Abbreviations: Asym, asymmetric; BSA, bovine serum albumin; CD, cytochalasin D; ChIP, chromatin immunoprecipitation; DAPI, 4'-6-diamido-2-phenylindole; DSC, distributed stem cell; H3K27me3, trimethylated histone H3 lysine 27; H3K4me3, trimethylated histone H4 lysine 4; ISIF, in situ immunofluorescence; MFI, mean fluorescence intensity; NGS, normal goat serum; PBS, phosphate-buffered saline; SACK, suppression of asymmetric cell kinetics; Sym, symmetric; Xn, xanthine Received 8.7.14; revised 19.10.14; accepted 30.10.14; Edited by G Raschellà
} 
denaturants that would confound investigations of molecular properties of immortal chromosomes that might illuminate their cellular function and components of the mechanism of nonrandom co-segregation. Recently, we reported the histone H2A variant H2A.Z as a pattern-specific, prospective biomarker for mouse immortal chromosomes. In both engineered mouse cell lines and mouse hair follicle DSCs undergoing nonrandom co-segregation, by immunofluorescence, H2A.Z is detected primarily on immortal chromosomes. The H2A.Z chromosomal asymmetry assay has $89 \%$ concordance with traditional DNA base analog assays for detection of immortal chromosomes. $^{6}$

The combination of SACK-expanded cell strains highly enriched for DSCs and H2A.Z as a native biomarker for immortal chromosomes is a technical advance that enables a new approach to investigation of the molecular basis of nonrandom chromosome co-segregation in DSCs. This approach is interrogation of DSCs for proteins and other labile cellular factors that are detected specifically on either immortal chromosomes or mortal chromosomes. Such distinctive patterns of detection could indicate specific structural or functional features of the mechanisms responsible for nonrandom co-segregation and associated asymmetric self-renewal by DSCs. Here, we report the first trial of this newly available strategy. We find that two well-described molecular marks of gene regulation, trimethylation of histone $\mathrm{H} 3$ lysines 27 and 4, display such an asymmetric distribution of detection between immortal chromosomes and mortal chromosomes in asymmetrically self-renewing mouse hair follicle-derived DSCs.

\section{Results}

Using H2A.Z asymmetry to identify immortal chromosomes in SACK-expanded mouse hair follicle DSCs, we applied specific monoclonal antibodies in indirect in situ immunofluorescence
(ISIF) analyses to investigate the respective chromosomal content of trimethylation on histone H3 lysine 27 (H3K27me3) and lysine 4 (H3K4me3). These epigenetic modifications were evaluated because of their reported roles in transcriptional suppression and activation, respectively. ${ }^{8}$ To insure the specificity of respective H2A.Z and H3K27me3 or H3K4me3 fluorescence signals, control analyses were performed that omitted each primary antibody individually or both together. In addition, antigen-blocking experiments were performed with respective peptide epitope antigen for H3K27me3 and H3K4me3 antibodies. The specificity of the H2A.Z antibodies was confirmed in this manner in an earlier study. ${ }^{6}$ Preincubation of the anti-H3K27me3 antibodies at room temperature for $1 \mathrm{~h}$ with $0.1 \mu \mathrm{g}$ of $\mathrm{H} 3 \mathrm{~K} 27 \mathrm{me} 3$ peptide epitope antigen ablated anti-H3K27me3-dependent fluorescence. Similarly, pre-incubation of the anti-H3K4me3 antibodies at room temperature for $1 \mathrm{~h}$ with $0.1 \mu \mathrm{g}$ of $\mathrm{H} 3 \mathrm{~K} 4 \mathrm{me} 3$ peptide epitope antigen ablated anti-H3K4me3-dependent fluorescence (Supplementary Fig. S1,Supplementary Information).

The previously described cytochalasin D (CD)-arrest assay was used for these studies, because of its advantage of exact sister-sister comparisons of nuclear proteins. ${ }^{6,7,10,11,26}$ Like H2A.Z, both epigenetic marks also showed examples of CDarrested binucleated cells with significant chromosomal asymmetry (Figure 1, Asym). On the basis of the control distribution of \%differences between the 4'-6-diamido-2-phenylindole (DAPI) mean fluorescence intensity (MFI) of sister nuclei in CD-arrested cells (See Figure 2c), for quantitative analysis, chromosomal asymmetry was defined as a \%difference in MFI $>30 \%$. Reciprocally, a symmetric chromosomal detection pattern was defined as $\leqslant 30 \%$ difference.

CD-arrested cells that displayed H3K27me3 asymmetry or H3K4me3 asymmetry also had a high rate of associated H2A.Z chromosomal asymmetry (Figure 1, Asym; Figure 2, compare quadrant 2 for $a, b$, and $d$ with $c$ ). The frequencies of a

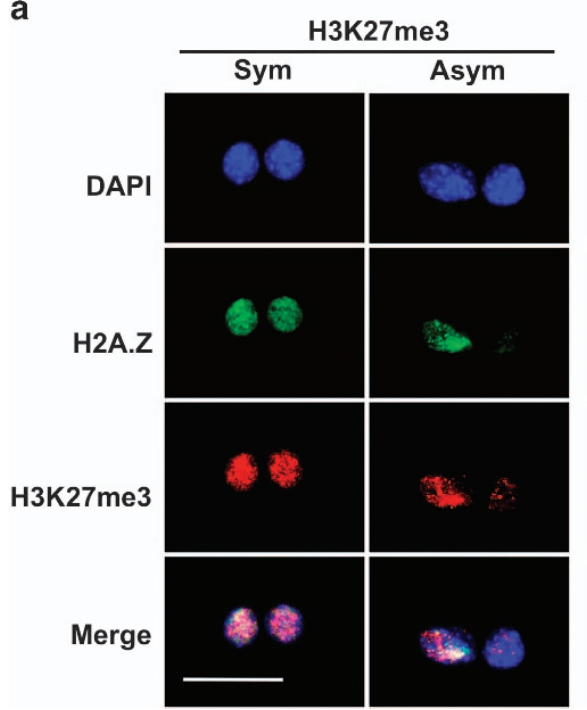

b

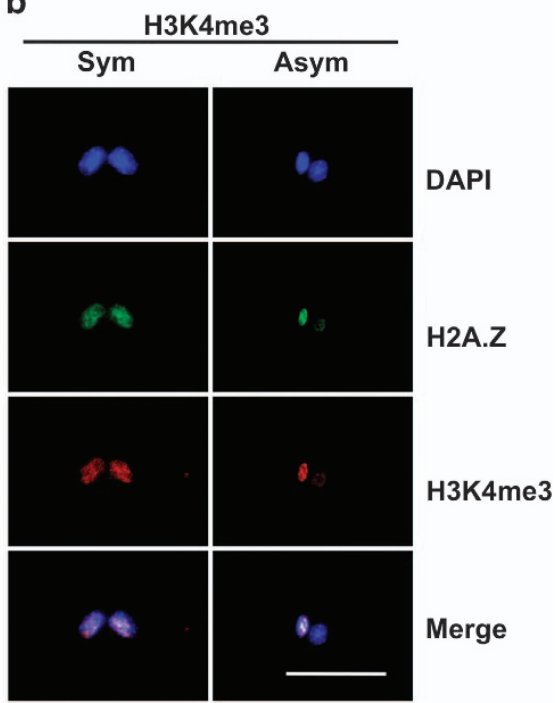

Figure 1 Respective association of chromosomal H3K27me3 and H3K4me3 detection level with H2A.Z-positive immortal chromosomes in murine hair follicle DSCs undergoing nonrandom co-segregation. Shown are examples of CD-arrested binucleated $3 \mathrm{C} 5$ cells, grown under conditions that promote nonrandom co-segregation, illustrating either symmetric (Sym) or asymmetric (Asym) detection of chromosomal H2A.Z by ISIF. (a) Examples of co-symmetric and co-asymmetric association of H3K27me3 detection level (b). Examples of co-symmetric and co-asymmetric association of H3K4me3 detection level. DAPI, nuclear DNA fluorescence. Merge, overlay of above three fluorescence images. Scale bar $=25$ microns 


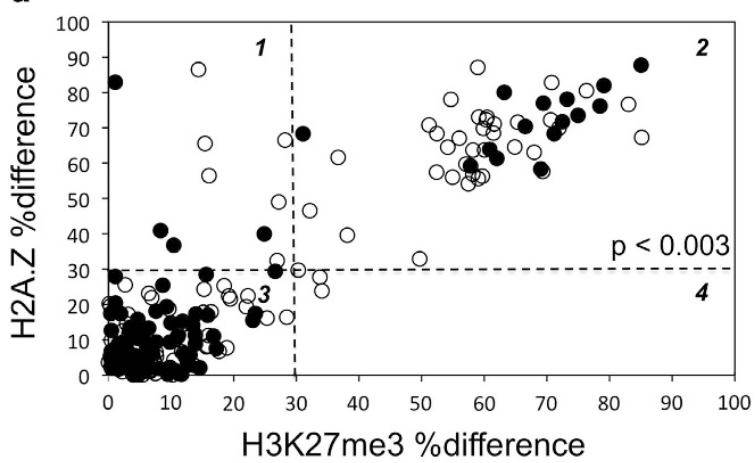

C

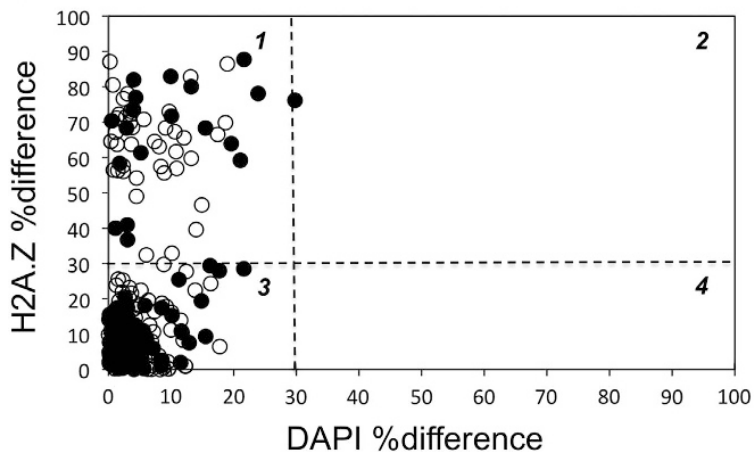

b

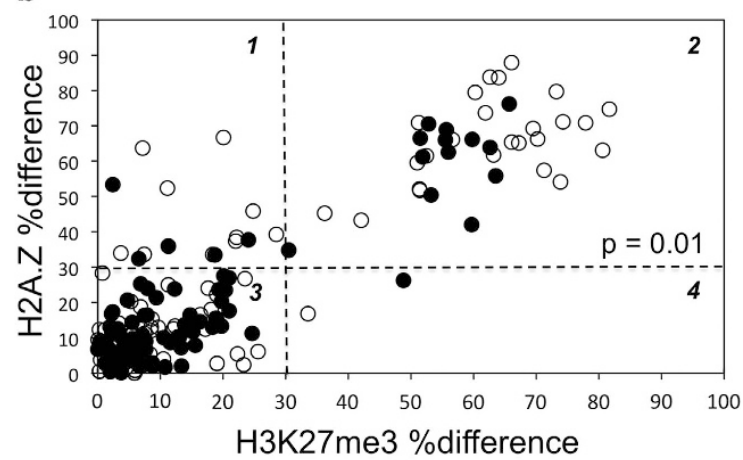

d

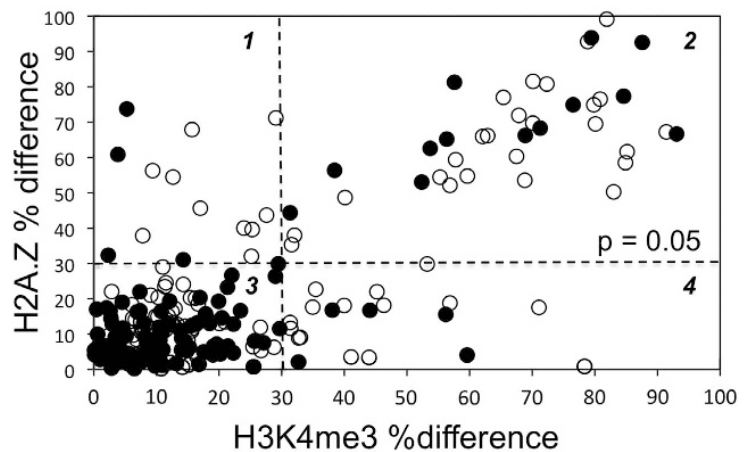

Figure 2 Quantitative analyses of the associated detection of H2A.Z chromosomal asymmetry with respective H3K27me3 and H3K4me3 chromosomal asymmetry during nonrandom co-segregation. Strain 3C5 (a, c, d) and strain 5B8 (b) murine hair follicle DSCs were evaluated under conditions that either promoted (Xn-free; open circles) or suppressed (Xn-supplemented; closed circles) nonrandom co-segregation. After CD-arrest, binucleated cells were evaluated by quantitative ISIF with anti-H2A.Z antibodies in combination with respective anti-H3K27me3 antibodies (a, b) or anti-H3K4me3 antibodies (d). The percent difference in mean fluorescence intensity (\%difference) for H2A.Z detection between sister nuclei in each examined binucleated cell is plotted against the corresponding \%difference for either $\mathrm{H} 3 \mathrm{~K} 27 \mathrm{me} 3$ detection (a, b) or $\mathrm{H} 34 \mathrm{Kme} 3$ detection (d). In c, the corresponding distribution of the \%difference in DAPI mean fluorescence intensity for the same binucleated cells evaluated in a was use to set thresholds for chromosomal detection patterns. Based on this analysis, a \%difference $\leqslant 30 \%$ was used to define a symmetric chromosomal detection pattern; and a \%difference $>30 \%$ defined an asymmetric chromosomal detection pattern. Quadrant 2 data correspond to binucleated cells exhibiting dual asymmetry. $P$-values indicate the level of statistical confidence, from the two-tailed Fisher's exact test, that the observed reduction in binucleated cells with dual asymmetry under conditions that suppress nonrandom co-segregation is not a chance occurrence

both types of cells with dual chromosomal asymmetry were significantly reduced by $\mathrm{Xn}$ supplementation. These properties are indicative of a dependency on nonrandom co-segregation, which is highly associated with H2A.Z asymmetry ${ }^{6}$ and suppressed by the guanine ribonucleotide precursor $\mathrm{Xn}^{7,11}$ Under $\mathrm{Xn}$-free conditions, which foster nonrandom co-segregation, strains 3C5 and 5B8 cells had respective $32 \%$ (Figure $2 a$, quadrant 2 , open circles) and $30 \%$ (Figure 2b, quadrant 2, open circles) frequencies of $C D$ arrested cells displaying both $\mathrm{H} 3 \mathrm{~K} 27 \mathrm{me} 3$ asymmetry and H2A.Z asymmetry. Xn-supplementation reduced these frequencies to $15 \%$ (Figure $2 \mathrm{a}$, quadrant 2 , closed circles) and $13 \%$ (Figure $2 \mathrm{~b}$, quadrant 2 , closed circles), respectively.

To a lesser but significant extent, examination of strain $3 \mathrm{C5}$ revealed that it also produced cells with associated $\mathrm{H} 3 \mathrm{~K} 4 \mathrm{me} 3$ and $\mathrm{H} 2 \mathrm{~A} . \mathrm{Z}$ asymmetry (Figure $2 \mathrm{~d}$, quadrant 2 ). Cells with this feature decreased from $21 \%$ (Figure 2d, quadrant 2, open circles) to $11 \%$ (Figure $2 \mathrm{~d}$, quadrant 2 , closed circles) when cultures were cultured in Xn-supplemented medium. We confirmed that the observed dual marker asymmetry was not due to interference between co-evaluating antibodies. In the case of H2A.Z and H3K27me3 dual asymmetry, we confirmed that the frequency of $3 \mathrm{C} 5$ DSCs with H3K27me3 asymmetry was similar when the anti-H3K27me3 antibodies were used alone (25\%) or in combination with anti-H2A.Z antibodies, whether they were bound first (as routinely; See Materials and Methods; 33\%) or second in the procedure $(26 \%)$.

Under Xn-free conditions, which maximize nonrandom cosegregation, $97 \%$ of cells showing H3K27me3 asymmetry also showed H2A.Z asymmetry (Figures $2 \mathrm{a}$ and b, compare quadrant 2 with quadrant 4 ); and $62 \%$ of cells exhibiting H3K4me3 asymmetry also showed H2A.Z asymmetry (Figure 2d, compare quadrant 2 with quadrant 4). Direct inspection of ISIF images (as in Figure 1, Asym) demonstrated that essentially all binucleated cells with both H2A.Z asymmetry and respective $\mathrm{H} 3$ trimethylation asymmetry were 'coasymmetric.' Quantitatively, the H2A.Z-positive immortal chromosome set showed elevated H3K27me3 and elevated H3K4me3 detection, respectively, compared with the opposing H2A.Z-negative chromosome set. In contrast, binucleated cells with configurations of symmetric $\mathrm{H} 2 \mathrm{~A}$.Z:asymmetric $\mathrm{H} 3$ trimethylation (Figures 2a, b and d, quadrant 4) and asymmetric H2A.Z:symmetric $\mathrm{H} 3$ trimethylation (Figures $2 \mathrm{a}$, b and d, quadrant 1) were infrequent; and anti-asymmetric binucleated cells (that is, with H2A.Z-positive immortal 
chromosomes, but elevated trimethylation detection on mortal chromosomes) were not observed.

By transitive logic, binucleated cells showing H2A.Z: H3K27me3 co-asymmetry and H2A.Z:H3K4me3 coasymmetry must also be co-asymmetric for $\mathrm{H} 3 \mathrm{~K} 27 \mathrm{me} 3$ and H3K4me3. On the basis of quantitative analyses presented in Figure 2, we estimate that essentially all cells undergoing nonrandom co-segregation are co-asymmetric for elevated H2A.Z and H3K37me3 detection on immortal chromosomes; and at least $\sim 60 \%$ of these binucleated cells are also coasymmetric for elevated $\mathrm{H} 3 \mathrm{~K} 4 \mathrm{me} 3$ detection on immortal chromosomes as well.

To confirm that H3K27Kme3 asymmetry and H3K4me3 asymmetry were due to lower detection on mortal chromosomes compared with levels on randomly segregated chromosomes in symmetrically self-renewing cells, we evaluated the total MFI of binucleated cells with respect to their \%difference in MFI (Figure 3). Similar to H2A.Z, which we reported earlier to have reduced detection on mortal chromosomes ${ }^{6}$ (Figure $3 a$ ), binucleated cells with H3K27me3 asymmetry and H3K4me3 asymmetry exhibited a significantly lower total MFI (Figures $3 \mathrm{~b}$ and c, respectively). Respectively, the average MFI of binucleated cells showing asymmetric chromosomal detection decreased significantly by 49,38 , and $23 \%$ (Figures $3 a-c$, respectively; $>30 \%$ difference) compared with binucleated cells showing symmetric chromosomal detection (Figure 3, $\leqslant 30 \%$ difference). Figure 4 data show that the reduced average level of detection is accounted for by the lower detection of H3K27me3 (Figure 4a) and H2K4me3 (Figure 4b) specifically on one set of chromosomes, which correspond to the mortal chromosome set (that is, H2A.Z-negative); whereas the immortal chromosomes (that is, H2A.Z-positive) retain a detection level comparable to randomly segregated chromosomes.

\section{Discussion}

The observed global co-asymmetry of increased H3K27me3 and H3K4me3 detection on immortal chromosomes is reminiscent of the gene-specific bivalency of these epigenetic marks that have been reported for embryonic cells, ${ }^{27}$ embryonic stem cells, ${ }^{8,9,27,28}$ and DSCs, including hematopoietic DSCs, ${ }^{29}$ neural DSCs, ${ }^{9}$ and primordial germ DSCs. ${ }^{30}$ In particular, this bivalent state is considered to hold in check transcription of genes involved in the developmental regulation and tissue-specific cell differentiation that are 'poised' in stem cells for initiation of developmental programs that occur with conversion to monovalent states. The global reduction of H3K27me3 and H3K4me3 detection on mortal chromosomes in nonrandomly co-segregating mouse hair follicle DSCs may reflect a similar, except asymmetric, activation of previously bivalent genes that is restricted to the chromosomes that will segregate to lineage-committed sisters produced during asymmetric self-renewal by DSCs.

An important consideration for interpretations of all epigenomic studies using immunodetection is the possibility that failure to detect a given antigenic epitope could be due to either its physical absence or its molecular masking. Though usually unable to assess or overlooked, this qualification applies to all chromatin immunoprecipitation-based assays, as a

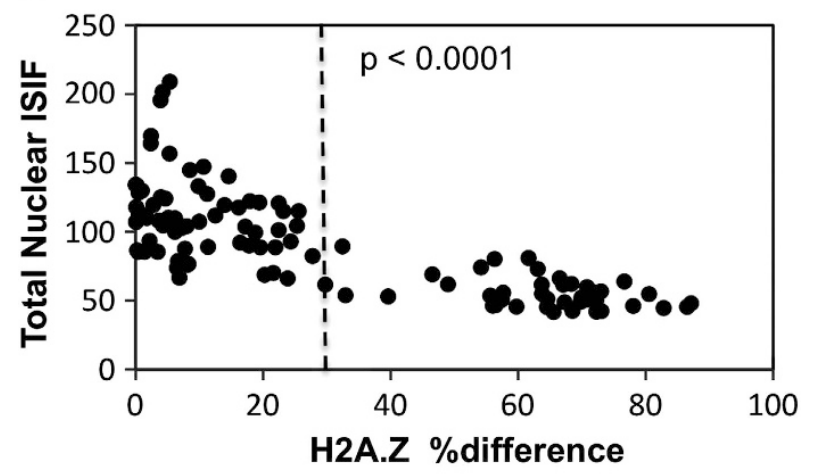

b

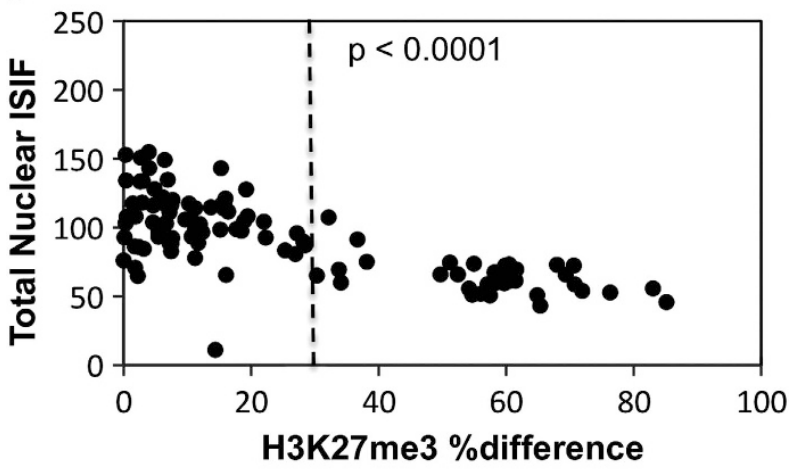

C

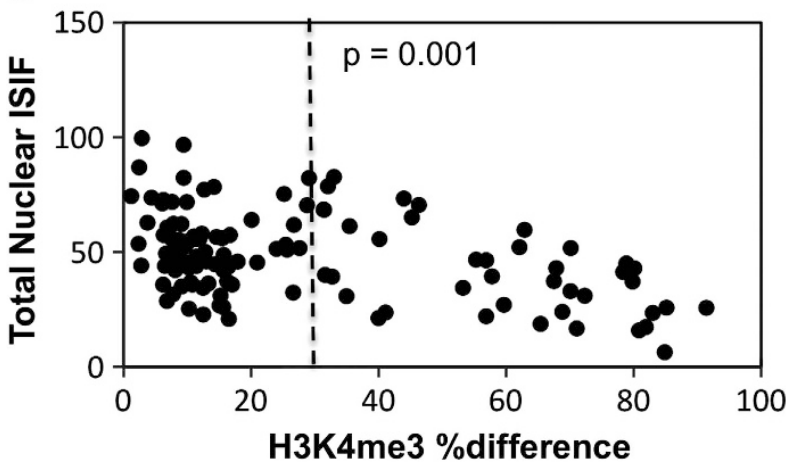

Figure 3 Murine hair follicle DSCs undergoing nonrandom co-segregation have a lower degree of $\mathrm{H} 3 \mathrm{~K} 27 \mathrm{me} 3$ and H3K4me3 detection. The total nuclear ISIF of strain $3 \mathrm{C} 5$ binucleated cells, described in Figure 2 for conditions that promote nonrandom co-segregation (Xn-free), was evaluated with respect to their corresponding \% difference in the mean fluorescence intensity of sister nuclei for (a), H2A.Z; (b), H3K27me3; and (c), H3K4me3. The vertical dotted line at 30\% difference demarcates the separation between binucleated cells with a symmetric (left of line) versus an asymmetric (right of line) chromosomal detection pattern, as described in the legend of Figure 2. $P$-values indicate the level of statistical confidence, from the Student's unpaired $t$-test, that the observed lower mean total nuclear fluorescence of asymmetric binucleated cells (\%difference $>30$ ), compared with symmetric binucleated cells (\%difference $\leqslant 30$ ), is not due to chance

well as the findings presented here. In fact, previously, we have found that H2A.Z chromosomal asymmetry during nonrandom co-segregation is due to molecular masking. ${ }^{6}$ In the present studies, so far, we have not identified mildly denaturing conditions that preserve H3K27me3 and H3K4me3 antigenic epitopes on immortal chromosomes, so that we can look for masking of the trimethylations on mortal 
a

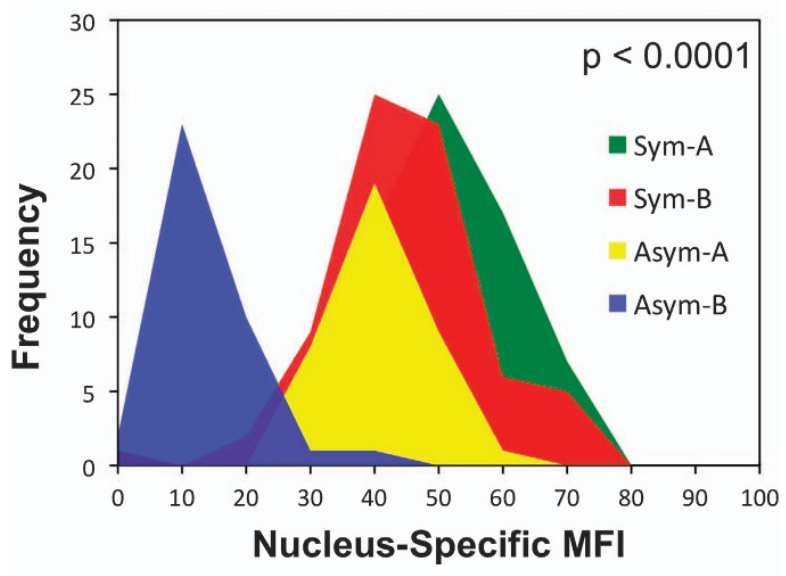

b

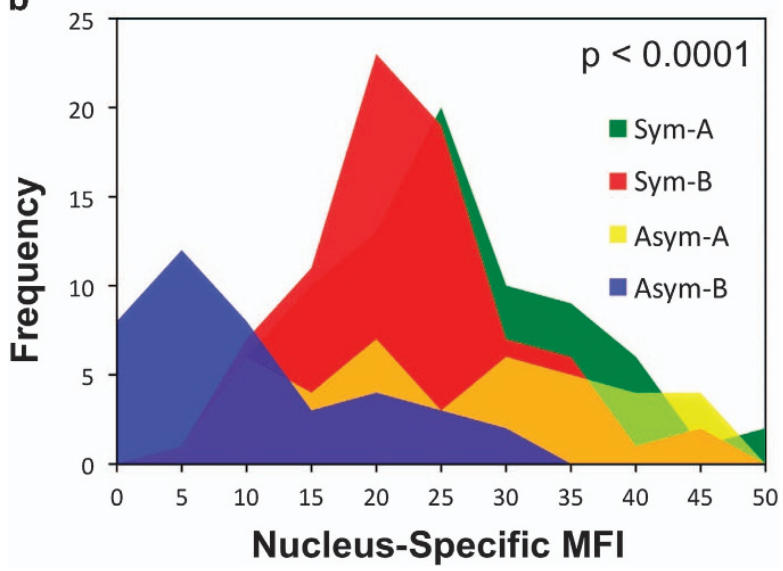

Figure 4 Mortal chromosomes in nonrandomly co-segregating murine hair follicle DSCs have a significantly reduced level of H3K27me3 and H3K4me3 detection. The mean fluorescence intensity (MFI) of four distinct groups of nuclei from the ISIF analyses of strain 3C5 binucleated cells, described in Figure 2 for conditions that promote nonrandom co-segregation (Xn-free), were used for these analyses. In each pair of nuclei in binucleated cells defined with either an asymmetric (Asym) or symmetric (Sym) chromosomal detection pattern, the nucleus with the greater MFI was designated as the ' $A$ ' nucleus; and the nucleus with lesser MFI, as the ' $B$ ' nucleus. The MFI distributions for each respective nuclear type (nuclear-specific MFI; Sym-A, Sym-B, Asym-A, Asym-B) are overlaid for comparison. (a) analysis for H3K27me3; (b) analysis of H3K4me3. P-values indicate the level of statistical confidence, from the Student's unpaired $t$-test, that the observed lower mean of the MFI distribution of Asym-B nuclei, compared with Sym-B nuclei, is not due to chance

chromosomes. Therefore, a molecular masking mechanism cannot be excluded at this time. However, whether the epigenetic marks are reduced or masked, our findings reveal a significant new property of nonrandomly co-segregating chromosomes in DSCs that is likely to manifest mechanisms of asymmetric DSC fate specification and/or mechanisms of nonrandom co-segregation. In addition, the co-elevation of H3K27me3 and H3K4me3 on the chromosomes of asymmetrically self-renewing DSCs, compared with their lineagecommitted sisters, may constitute a new specific biomarker for DSC identification in tissues.

An important issue that this first study cannot address is whether the findings apply to DSCs in tissues in general. There have been several reports of failure to detect nonrandom co-segregation in vitro cell populations enriched for specific stem types ${ }^{31}$ or in specific stem cell populations in vivo. ${ }^{32-35}$ In the latter case, reported in vivo investigations of mouse skin hair follicles ${ }^{32,33}$ were contradicted by the expansion of cells from mouse whisker follicles, which exhibit nonrandom co-segregation in cell culture. ${ }^{7}$ We have commented previously on possible technical explanations for these and other discrepancies ${ }^{7,36}$ (ref. 35, online comment).

As a biological consideration, there is still quite limited knowledge of how mammalian DSC maintenance programs may differ among different tissues. On the basis of our ex vivo studies, we have proposed that deterministic asymmetric selfrenewal and associated nonrandom co-segregation predominate in vivo. ${ }^{37}$ During deterministic asymmetric self-renewal, individual DSCs divide asymmetrically. However, this hypothesis is at odds with a growing body of work that is consistent with the view that neutral competition is the cellular basis for DSC asymmetric self-renewal in tissues. ${ }^{38}$ When it has been possible to define the asymmetric self-renewal pattern of DSCs undergoing nonrandom co-segregation, it has been deterministic. ${ }^{7}$ Asymmetric self-renewal by neutral competition does not require that individual DSCs divide asymmetrically. Theoretically, with neutral competition, nonrandom co-segregation would be ineffective for preserving DSC genetic fidelity. ${ }^{37}$ However, it could still be effective in determining DSC's fate stochastically, as is required for DSC compartment maintenance by neutral competition. ${ }^{38}$

Here, it is important to emphasize that experimental data consistent with neutral competition, though very well developed, cannot and does not exclude deterministic asymmetric self-renewal by DSCs. ${ }^{38,39}$ So, the many examples of nonrandom co-segregation detected in vivo, ex vivo, and in vitro could occur in tissues maintained by neutral competition or deterministic asymmetric self-renewal. If neutral competition is responsible, the asymmetric reduction in the detection of H3K27me3 and H3K4me3 on mortal chromosomes could signify a function in stochastic DSC fate determination. If deterministic asymmetric self-renewal is responsible, the reduction could indicate important aspects of mechanisms for DSC fate determination and genetic fidelity. We favor the latter explanation as a universal feature of DSCs in the majority of postnatal mammalian tissues. ${ }^{37}$

\section{Materials and methods}

Mouse hair follicle DSCs were maintained as previously described. ${ }^{7}$ Cells were fixed at room temperature with $3.7 \%$ paraformaldehyde/phosphate-buffered saline (PBS) and permeabilized with $0.2 \%$ Triton X-100/PBS for 10 min each, followed by blocking with $10 \%$ normal goat serum in PBS normal goat serum (NGS). Blocked cells were incubated with mouse anti-H3K27me3 monoclonal antibodies (Cat\# ab6002, Abcam, Cambridge, MA, USA; diluted 1: 100 in 10\% NGS, $1 \mathrm{~h}$, room temperature) or mouse anti-H3K4me3 monoclonal antibodies (Cat\# ab12209, Abcam; diluted $1: 200$ in 10\% NGS, $16-24 \mathrm{~h}, 4^{\circ} \mathrm{C}$ ). Thereafter, slides were washed with $0.5 \%$ bovine serum albumin (BSA) in PBS and incubated at room temperature for $1 \mathrm{~h}$ with Alexa Fluor $568 \mathrm{~nm}$-conjugated secondary goat anti-mouse IgG antibodies (Cat\# A-11004, Invitrogen, Inc., Carlsbad, CA, USA) diluted 1:500 in 10\% NGS. Thereafter, slides were washed with $0.5 \% \mathrm{BSA}$ and incubated for $16-24 \mathrm{~h}$ at $4{ }^{\circ} \mathrm{C}$ with rabbit anti-H2A.Z polyclonal antibodies (Cat\# 2718, Cell Signaling Technology, Danvers, MA, USA) diluted $1: 200$ in 10\% NGS. Slides were then washed with $0.5 \%$ BSA and incubated at room temperature for $1 \mathrm{~h}$ with Alexa Fluor 488-conjugated secondary goat antirabbit IgG antibodies (Cat\# A-11008, Invitrogen, Inc.) diluted 1:300 in 10\% NGS. Continuing, slides were washed with $0.5 \% \mathrm{BSA}$ and mounted with DAPI-containing VectaShield mounting media. Peptide epitope antigens for specificity blocking 
experiments were purchased from Abcam (H3K27me3 peptide: Cat\# ab1782; H3K4me3 peptide: Cat\# ab1342). Epifluorescence images were captured with a Leica DMR microscope and Leica DC300F digital camera system. The mean pixel intensity of fluorescent nuclei was quantified using NIH Image $\mathrm{J}$ software. ${ }^{6,7}$ The $\%$ difference in MFI between sister nuclei was calculated as $((A-B) / A) \times 100 \%$, where $A \geqslant B$. Total nuclear fluorescence equals $A+B$. The statistical significance of observed differences in categorical data was evaluated in $2 \times 2$ contingency tables using Fisher's two-tailed exact test. Student's $t$-test was used to evaluate the statistical significance of differences in the MFI of compared groups of nuclei.

\section{Conflict of Interest}

Dr Huh declares no conflict of interest. Dr Sherley is the sole proprietor of the Adult Stem Cell Technology Center, LLC, a for-profit company pursuing commercialization of technologies for the detection and quantification of distributed stem cells.

Acknowledgements. The research was supported by NIH-NIGMS Director's Pioneer Award \#5DP10D000805 and a grant from the Lee lacocca Family Foundation.

\section{Author contributions}

JLS was responsible for the conception and design of the experiments, data analyses and statistical analyses, the interpretation of the results, and writing the manuscript. YHH was responsible for the conception and design of the experiments and performed all the experiments reported. YHH also was responsible for cell imaging, data analyses and statistical analyses, the interpretation of the results, and writing the manuscript.

1. Cairns J. Mutation selection and the natural history of cancer. Nature 1975; 255: 197-200.

2. Sherley JL. A new mechanism for aging: chemical 'age spots' in immortal DNA strands in distributed stem cells. Breast Disease 2008; 29: 37-46.

3. Taghizadeh RR, Sherley JL. Expanding the therapeutic potential of umbilical cord blood hematopoietic stem cells. In: Cetrulo CL, Cetrulo KJ, Cetrulo Jr CL (eds). Perinatal stem cells. Wiley-Blackwell: Hoboken, 2009, pp 21-40

4. Lee H-S, Crane GG, Merok JR, Tunstead JR, Hatch NL, Panchalingam K et al. Clonal expansion of adult rat hepatic stem cell lines by suppression of asymmetric cell kinetics (SACK). Biotech Bioeng 2003; 83: 760-771.

5. Paré J-F, Sherley JL. Biological principles for ex vivo adult stem cell expansion In: Schatten G (ed), Current topics in developmental biology. Vol. 73. Elsevier, Inc.: San Diego, 2006, pp 141-171

6. Huh YH, Sherley JL. Molecular cloaking of H2A.Z on mortal DNA chromosomes during nonrandom segregation. Stem Cells 2011; 29: 1620-1627.

7. Huh YH, King J, Cohen J, Sherley JL. SACK-expanded hair follicle stem cells display asymmetric nuclear Lgr5 expression with non-random sister chromatid segregation. Sci Rep 2011; 1: 175

8. Voigt P, Tee WW, Reinberg D. A double take on bivalent promoters. Genes Dev. 2013; 27: 1318-1338.

9. Mikkelson TJ. Genome-wide maps of chromatin state in pluripotent and lineagecommitted cells. Nature 2007; 448: 553-562.

10. Merok JR, Lansita JA, Tunstead JR, Sherley JL. Cosegregation of chromosomes containing immortal DNA strands in cells that cycle with asymmetric stem cell kinetics. Cancer Res. 2002: 62: 6791-6795.

11. Rambhatla L, Ram-Mohan S, Cheng JJ, Sherley JL. Immortal DNA strand co-segregation requires p53/MPDH-dependent asymmetric self-renewal associated with adult stem cells. Cancer Research 2005; 65: 3155-3161.

12. Lark KG, Consigli RA, Minocha HC. Segregation of sister chromatids in mammalian cells. Science 1966; 154: 1202-1205.

13. Potten CS, Owen G, Booth D. Intestinal stem cells protect their genomes by selective segregation of template DNA strands. J Cell Sci 2002; 115: 2381-2388.

14. Karpowicz P, Morshead C, Kam A, Jervis E, Ramunas J, Cheng V et al. Support for the immortal strand hypothesis: neural stem cells partition DNA asymmetrically in vitro. J Cell Biol 2005; 170: 721-732.

15. Smith G. Label-retaining epithelial cells in mouse mammary gland divide asymmetrically and retain their template DNA strands. Development 2005; 132: 681-687.

16. Armakolas A, Klar AJS. Cell type regulates selective segregation of mouse chromosome 7 DNA strands in mitosis. Science 2006; 311: 1146-1149.
17. Shinin V, Gayraud-Morel B, Gomès D, Tajbakhsh S. Asymmetric division and cosegregation of template DNA strands in adult muscle satellite cells. Nat Cell Biol 2006; 8: 677-687.

18. Conboy MJ, Karasov AO, Rando TA. High incidence of non-random template strand segregation and asymmetric fate determination in dividing stem cells and their progeny. PLOS Biol 2007; 5: 1120-1126.

19. Capuco AV. Identification of putative bovine mammary epithelial stem cells by their retention of labeled DNA strands. Exp Biol Med 2007; 232: 1381-1390.

20. Pine SR, Ryan BM, Varticovski L, Robles Al, Harris CC. Microenvironmental modulation of asymmetric cell division in human lung cancer cells. Proc Natl Acad Sci USA 2010; 107: 2195-2200.

21. Hari H, Xin HW, Jaiswal K, Wiegand G, Kim BK, Ambe $\mathrm{C}$ et al. Isolation of live labelretaining cells and cells undergoing asymmetric cell division via nonrandom chromosoma cosegregation from human cancers. Stem Cells Devel 2011; 20: 1649-1658.

22. Sundararaman B, Avitabile D, Konstandin MH, Cottage CT, Gude N, Sussman MA. Asymmetric chromatid segregation in cardiac progenitor cells is enhanced by Pim-1 kinase. Circ Res 2012; 110: 1169-1173.

23. Kaistura J, Bai Y, Cappetta D, Kim J, Arranto C, Sanada F et al. Tracking chromatid segregation to identify human cardiac stem cells that regenerate extensively the infracted myocardium. Circ Res 2012; 111: 894-906.

24. Xin H-W, Hari DM, Mullinax JE, Ambe CM, Koizumi T, Ray S et al. Tumor-initiating labelretaining cancer cells in human gastrointestinal cancers undergo asymmetric cell division. Stem Cells 2012; 30: 591-598.

25. Lansdorp PM. Immortal strands? Give me a break. Cell 2007; 129: 1244-1247.

26. Noh M, Smith JL, Huh YH, Sherley JL. A resource for discovering specific and universal biomarkers for distributed stem cells. PLoS One 2011; 6(7): e22077.

27. Dahl JA, Reiner AH, Klungland A, Wakayama T, Collas P. Histone H3 lysine 27 methylation asymmetry on developmentally-regulated promoters distinguish the first two lineages in mouse preimplantation embryos. PLoS One 2010; 5(2): e9150.

28. Bernstein BE, Mikkelsen TS, Xie X, Kamal M, Huebert DJ, Cuff J et al. A bivalent chromatin structure marks key developmental genes in embryonic stem cells. Cell 2006; 125: 315-326.

29. Abraham BJ, Cui K, Tang Q, Zhao K. Dynamics regulation of epigenomic landscapes during hematopoiesis. BMC Genomics 2013; 14: 193-207.

30. Sachs M, Onodera C, Blaschke K, Ebata KT, Song JS, Ramalho-Santos M. Bivalent chromatin marks developmental regulatory genes in the mouse embryonic germline in vivo. Cell Rep 2013; 3: 1777-1784.

31. Kiel MJ, He S, Ashkenazi R, Gentry SN, Teta M, Kushner JA et al. Haematopoietic stem cells do not asymmetrically segregate chromosomes or retain BrdU. Nature 2007; 449: 238-242.

32. Sotiropoulou PA, Candi A, Blanpain C. The majority of multipotent epidermal stem cells do not protect their genome by asymmetrical chromosome segregation. Stem Cells 2008; 26: 2964-2973.

33. Waghmare SK, Bansal R, Lee J, Zhang YV, McDermitt DJ, Tumbar T. Quantitative proliferation dynamics and random chromosome segregation of hair follicle stem cells. EMBO J 2008; 27: 1309-1319.

34. Escobar M, Nicolas P, Sangar F, Laurent-Chabalier S, Clair P, Joubert D et al. Intestinal epithelial stem cells do not protect their genome by asymmetric chromosome segregation. Nat Commun 2010; 2: 258-266.

35. Steinhauser ML, Bailey AP, Senyo SE, Guillermier C, Perlstein TS, Gould AP et al. Multiisotope imaging mass spectrometry quantifies stem cell division and metabolism. Nature 2012; 481: 516-520.

36. Sherley JL. Overlooked areas need attention for sound evaluation of DNA strand inheritance patterns in Drosophila male germline stem cells. J Cell Sci 2011; 124: 4137.

37. Sherley JL. New cancer diagnostics and therapeutics from the ninth 'hallmark of cancer': symmetric self-renewal by mutated distributed stem cells. Expert Rev Mol Diagn 2013; 13: 797-810.

38. Klein AM, Simons BD. Universal patterns of stem cell fate in cycling adult tissues. Development 2011; 138: 3103-3111.

39. Snippert HJ, van der Flier LG, Sato T, van Es JH, van den Born M, Kroon-Veenboer C et al. Intestinal crypt homeostasis results from neutral competition between symmetrically dividing Lgr5 stem cells. Cell 2010; 143: 134-144.

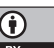

Cell Death and Disease is an open-access journal published by Nature Publishing Group. This work is licensed under a Creative Commons Attribution 3.0 Unported License. The images or other third party material in this article are included in the article's Creative Commons license, unless indicated otherwise in the credit line; if the material is not included under the Creative Commons license, users will need to obtain permission from the license holder to reproduce the material. To view a copy of this license, visit http:/ creativecommons.org/licenses/by/3.0/ 